\title{
Approximation hardness of Travelling Salesman via weighted amplifiers
}

\author{
Miroslav Chlebík ${ }^{1}$ and Janka Chlebíkováa \\ 1 Department of Mathematics, University of Sussex, United Kingdom, \\ m.chlebik@sussex.ac.uk \\ 2 School of Computing, University of Portsmouth, United Kingdom, \\ janka.chlebikova@port.ac.uk
}

\begin{abstract}
The expander graph constructions and their variants are the main tool used in gap preserving reductions to prove approximation lower bounds of combinatorial optimisation problems. In this paper we introduce the weighted amplifiers and weighted low occurrence of CONSTRAint SATISFACTION problems as intermediate steps in the NP-hard gap reductions. Allowing the weights in intermediate problems is rather natural for the edge-weighted problems as Travelling Salesman or Steiner Tree. We demonstrate the technique for Travelling SalesMAN and use the parametrised weighted amplifiers in the gap reductions to allow more flexibility in fine-tuning their expanding parameters. The purpose of this paper is to point out effectiveness of these ideas, rather than to optimise the expander's parameters. Nevertheless, we show that already slight improvement of known expander values modestly improve the current best approximation hardness value for TSP from $\frac{123}{122}([9])$ to $\frac{117}{116}$. This provides a new motivation for study of expanding properties of random graphs in order to improve approximation lower bounds of TSP and other edge-weighted optimisation problems.
\end{abstract}

\section{Introduction}

The Travelling Salesman problem (TSP) is undoubtedly one of the most famous combinatorial optimisation problems. In its standard version, we are given an edge-weighted (undirected) graph and the goal is to find a closed tour with a minimum cost that visits each vertex at least once. This is equivalent to the Graphic Travelling Salesman problem where exactly one visit per vertex is allowed and the cost between any two vertices corresponds to their shortest path.

The shortest-path metric of the GRAPHIC TSP plays an important role in understanding of complexity for the METRIC TSP problem. The approximability of the METRIC TSP is a long-standing open problem, Christofides's approximation algorithm with ratio 1.5 [4] hasn't been improved for more than three decades. It is generally believed that the approximation ratio can be close to $4 / 3$ due to known integrality gap for the Held-Karp LP relaxation [8].

In the last decade, some significant progress has been done in the GRAPHIC TSP. Gharan et al. [6] made first breakthrough with an $(1.5-\varepsilon)$-approximation 
algorithm where $\varepsilon$ being of the order of $10^{-12}$. Following that, Mömke and Svensson [11] obtained a significantly better approximation factor of $\frac{14(\sqrt{2}-1)}{12 \sqrt{2}-13} \approx$ 1.461 , which was improved further to $\frac{13}{9} \approx 1.444$ by Mucha [12]. To our best knowledge, currently the best known approximation ratio is 1.4 due to [15]. The overview about this recent development can also be found in [16].

However, there is still a significant gap between the ratio of the best approximation algorithm and the approximation ratio that provably can't be achieved unless $\mathrm{P}=\mathrm{NP}$. The first APX-hardness result showed the NP-hardness to approximate the TSP problem within $1+\varepsilon$ without any explicit value for $\varepsilon$ (Papadimitrious and Yannakakis, [13]). The first explicit value 5381/5380 was set by Engebretsen [5], further improved to 3813/3812 by Böckenhauser et al. [1] and 220/219 by Papadimitrious and Vempala ([14]). The further progress in the reductions and amplifiers increased the threshold to $185 / 184$ by Lampis ([10]) and to our best knowledge the currently best value is $123 / 122$ by Karpinski et al. $([9])$.

Main contribution. The main novelty of this paper is using weighted amplifiers and weighted low occurrence of CONSTRAint SATISFACTION problems (CSP) as intermediate steps in the NP-hard gap reductions to the Travelling SAlESMAN problem. Allowing the weights in intermediate problems to TSP (or the SteINER TREE problem) is rather natural, as the problems themselves are using edge weights. We demonstrate the technique for TSP and use the parametrised weighted amplifiers in the gap reductions to allow more flexibility in fine-tuning their expanding parameters. In this paper we don't aim to optimise the parameters of amplifiers that provably exist, but show that already slight improvement of known values modestly improve the hardness of approximation for TSP from the current best value $\frac{123}{122}([9])$ to the new value $\frac{117}{116}$. This provides a new motivation for study of expanding properties of random graphs in order to improve approximation lower bounds of TSP and other edge-weighted optimisation problems.

\section{Preliminaries}

All graphs in this paper are undirected and connected. Let $G=(V, E)$ be an edge-weighted graph with cost edge-function $c: E \rightarrow \mathbb{R}^{+}$. For an edge $e=$ $\{u, v\} \in E$ we also use the notation $u v$ as an shorthand. A tour in the graph $G$ is an alternating sequence of vertices and edges, starting and ending at a vertex, where each vertex is incident with the previous and the following edge in the sequence. If a starting and ending vertex is the same, the tour is closed.

Any solution of TSP is a closed tour, hence an Eulerian multigraph (edges are taken with their multiplicities if they are used multiple times) spanning $V$. A quasi-tour $T$ in $G$ is a multiset of edges from $E$ such that all vertices in $G$ are balanced with respect to $T$ (each vertex from $V$ is incident with even number of edges from $T$, possibly 0 ); hence each such connected component in $G$ is an Eulerian multigraph (or an isolated vertex). 


\section{MAX-E3-LIN-2}

Our inapproximability results for the TRAVELLing SALESMAn problem use reductions from Håstad's NP-hard gap type result for MAX-E3-LIN-2, the MAXImUM SATISFIABILITY problem for linear equations modulo 2 with exactly 3 variables per equation [7] (more details can be found in Appendix). In fact, Håstad's tight inapproximability results can be stated in the form in which every variable occurs the same number of times in the system of equations, see e.g. [2].

Theorem 1. For every $\varepsilon \in\left(0, \frac{1}{4}\right)$ and every fixed sufficiently large integer $k \geq k(\varepsilon)$, the following partial decision subproblem $Q(\varepsilon, k)$ of MAX-E3-LIN-2 is NP-hard: given an instance of MAX-E3-LIN-2 with $m$ equations and exactly $k$ occurrences of each variable, to decide if at least $(1-\varepsilon) m$ or at most $\left(\frac{1}{2}+\varepsilon\right) m$ equations are satisfied by the optimal assignment.

The results of such form were already used to prove the inapproximability results for other optimisation problems, e.g., the STEINER TREE problem [3].

For some optimisation problems it is more convenient to use reductions if all equations of MAX-E3-LIN-2 have the same right hand side. The NP-hard gap results in such a case can be easily enforced if we allow flipping some occurrences of variables, so also the literal $\bar{x}(:=1-x)$ can be used for a variable $x$. The canonical gap versions $Q_{b}(\varepsilon, 2 k)$, for any fixed $b=0$ or $b=1$, of MAX-E3-LIN-2 are as follows:

The $Q_{b}(\varepsilon, 2 k)$ PRoBlem, $b \in\{0,1\}$

Input: An instance of MAX-E3-LIN-2 with $m$ equations of the form $x \oplus y \oplus z=b$, each variable occurring exactly $k$ times as unnegated and $k$ times negated.

Task: To decide if at least $(1-\varepsilon) m$ or at most $\left(\frac{1}{2}+\varepsilon\right) m$ equations are satisfied by the optimal assignment.

The corresponding 'fixed occurrence' NP-hard gap result reads as follows (see [2] for the details of the following theorem):

Theorem 2. For every $\varepsilon \in\left(0, \frac{1}{4}\right)$ and every sufficiently large integer $k, k \geq$ $k(\varepsilon)$, the partial decision subproblems $Q_{0}(\varepsilon, 2 k)$ and $Q_{1}(\varepsilon, 2 k)$ of MAX-E3-LIN-2 are NP-hard.

\section{Weighted Amplifiers}

Amplifier graphs are useful in proving inapproximability results for CSPs in which every variable appears a bounded (and, typically, very low) number of times. Such CSPs are often used as intermediate steps in proving approximation hardness results for many combinatorial optimisation problems. For problems like Travelling Salesman, or Steiner Tree which are based on edge weights, it is natural to consider the intermediate low degree CSPs with their edge weights as well.

For a graph $G=(V, E)$, a cut is a partition of $V$ into two subsets $U$ and $\bar{U}:=V \backslash U$. The cut set $E(U, \bar{U})$ is defined as $E(U, \bar{U})=\{u v \in E, u \in$ $U$ and $v \in \bar{U}\}$ and the cut size as $|E(U, \bar{U})|$. If edges are weighted with $p: E \rightarrow$ $\mathbb{R}^{+}$, then $p(E(U, \bar{U}))$ is weight of the cut set $E(U, \bar{U})$, hence $p(E(U, \bar{U}))=$ $\sum_{u v \in E, u \in U, v \in \bar{U}} p(u v)$. 
Definition 1. Let $G=(V, E)$ be a graph with edge weights $p: E \rightarrow \mathbb{R}^{+}$, and $D \subseteq V,|D| \geq 2$. We say that a weighted graph $(G, p)$ is an amplifier for $D$ if for every vertex set $A \subseteq V$

$$
p(E(A, \bar{A})) \geq \min \{|D \cap A|,|D \cap \bar{A}|\} .
$$

The vertices of the given set $D$ are called the contacts, the rest of the vertices $(=V \backslash D)$ is the set of checkers. We say that an amplifier $(G, p)$ for the set $D$ is a $d$-regular amplifier if, additionally, all contacts have degree $(d-1)$ and all checkers have degree $d$ (in $G$ ).

In full generality, one could also allow distinct weights for vertices of $D$ to replace the sizes $|D \cap A|,|D \cap \bar{A}|$ with their weighted version, but for our purposes the vertices of $D$ are uniformly weighted each with weight 1 .

\section{Intermediate weighted CSPs}

In this section we extend the NP-hard gap results from a system of linear equations with exactly 3 variables to a low occurrence version of $w$-MAX-3-LIN-2, a weighted hybrid system of linear equations over $\mathbb{Z}_{2}$ with either 2 or 3 variables. Similarly to MAX-E3-LIN-2, the task of the $w$-MAX-3-LIN-2 problem is to find an assignment that maximizes weight of the satisfied equations in the hybrid system.

To prove the NP-hard gap results for the $w$-MAX-3-LIN-2 problem, we extend Håstad's results for MAX-E3-LIN-2 using the amplifiers defined in Section 1.

Reduction from $Q(\varepsilon, k)$ to $w$-MAX-3-LIN-2

Let $\varepsilon \in\left(0, \frac{1}{4}\right)$, and $k>0$ be an integer such that the problem $Q(\varepsilon, k)$ is NP-hard. Let an instance $I$ of $Q(\varepsilon, k)$ be given, denote by $\nu(I)$ the set of variables of $I$, $\nu:=|\nu(I)|$. Let's assume that $G=(V, E)$ with the edge weights $p: E \rightarrow \mathbb{R}^{+}$be an amplifier for a set $D \subseteq V$ with $|D|=k$.

Now we describe a gap preserving reduction from $Q(\varepsilon, k)$ to the $w$-MAX-3-LIN-2 problem with an amplifier $(G, p)$ as a parameter. The instance $I$ of $Q(\varepsilon, k)$ is transformed to a weighted hybrid instance $J$ of $w$-MAX-3-LIN-2.

- For each variable $x \in \nu(I)$ take a copy of the amplifier $(G, p)$, let $\left(G_{x}, p\right)$ denote that copy:

- Inside $\left(G_{x}, p\right)$ the vertices correspond to the variables in $J$ and each edge $v v^{\prime}$ represents the equation $v \oplus v^{\prime}=0$ with weight $p\left(v v^{\prime}\right)$ in $J$.

The contact vertices of $\left(G_{x}, p\right)$ represent $k$ occurrences of the variable $x$ in the equations of $I$. Distinct occurrences of a variable $x$ in $I$ are represented by the distinct contact vertices in $G_{x}$.

- Every equation $x \oplus y \oplus z=b$ from $I, b \in\{0,1\}$, also belongs to $J$ with weight 1.

Remark 1. Observe that the above reduction from an instance $I$ of $Q(\varepsilon, k)$ to an instance $J$ of $w$-MAX-3-LIN-2 preserves the NP-hard gap of $Q(\varepsilon, k)$. Indeed, there is a simple dependence of an optimal value for $J$ on that of $I$. 
In the following we show that if we look at these problems as Minimum UNSATISFIABILITY problems, where $\mathrm{OPT}^{\prime}$ is the corresponding minimum weight of unsatisfied equations over all assignments, then $\operatorname{OPT}^{\prime}(I)=\mathrm{OPT}^{\prime}(J)$. Clearly, any assignment to variables from $\nu(I)$ generate an assignment to variable of $J$ in a natural way; the value of a variable $x \in \nu(I)$ is assigned to all variables of $G_{x}$. Such assignments to variables of $J$ are called standard. Hence, obviously $\operatorname{OPT}^{\prime}(J) \leq \operatorname{OPT}^{\prime}(I)$.

The observation that the optimum $\mathrm{OPT}^{\prime}(J)$ is achieved on standard assignments is based on the amplifier's properties. Any assignment $\varphi$ to the variables of $J$ can be converted to a standard one in such a way that the weight of unsatisfied equations doesn't increase as follows: consider a variable $x$ from $\nu(I)$. Assign to all variables in $G_{x}$ the same value as it is assigned to the majority of contact vertices in $G_{x}$ by the assignment $\varphi$. The fact that $\left(G_{x}, p\right)$ is the amplifier ensures that the weight of unsatisfied equations in $J$ doesn't increase. Now if we repeat the same operation for each variable from $\nu(I)$, one after another, the result will be a standard assignment without increase of the weight of unsatisfied equations in $J$. Consequently, $\mathrm{OPT}^{\prime}(J)$ is achieved on the standard assignments. But for every standard assignment the weight of unsatisfied equations of $J$ is the same as the number of unsatisfied equations of $I$ by that assignment, hence $\operatorname{OPT}^{\prime}(I)=\operatorname{OPT}^{\prime}(J)$.

Reduction from $Q_{b}(\varepsilon, 2 k)$ to $w$-MAX-3-LIN-2

Now we slightly modify the previous reduction from $Q(\varepsilon, k)$ to deal with the instances of $Q_{b}(\varepsilon, 2 k)$ for any fixed $b=0$ or $b=1$.

Let $\varepsilon \in\left(0, \frac{1}{4}\right)$ and $k>0$ be an integer such that $Q_{b}(\varepsilon, 2 k)$ is NP-hard. Assume that $G=(V, E)$ with edge weights $p: E \rightarrow \mathbb{R}^{+}$is an amplifier for a set $D \subseteq V$ with $|D|=2 k$. Let $\left\{V^{u}, V^{n}\right\}$ be a partition of $V$ balanced in $D$, namely $\left|D \cap V^{u}\right|=\left|D \cap V^{n}\right|=k$. Denote further $G^{u}$ and $G^{n}$ the induced subgraph of $G$ with the vertex sets $V^{u}$ and $V^{n}$, respectively. In what follows we describe the reduction from $Q_{b}(\varepsilon, 2 k)$ to $w$-MAX-3-LIN-2 parametrised by an amplifier $(G, p)$ for $D \subseteq V$ with $|D|=2 k$ and with chosen balanced partition $\left\{V^{u}, V^{n}\right\}$ of $V$.

Let an instance $I$ of $Q_{b}(\varepsilon, 2 k)$ be given, $\nu(I)$ be the set of variables of $I$, $\nu=|\nu(I)|$.

- For each variable $x$ from $\nu(I)$ take a copy of an amplifier $(G, p)$, let $G_{x}$ denote such a copy.

- Any edge $v v^{\prime}$ inside either $G_{x}^{u}$ or $G_{x}^{n}$ represents the cycle equation $v \oplus v^{\prime}=0$ taken with weight $p\left(v v^{\prime}\right)$.

- Any edge between $v \in V_{x}^{u}$ and $v^{\prime} \in V_{x}^{n}$ in $G_{x}$ represents the matching equation $v \oplus v^{\prime}=1$ taken with weight $p\left(v v^{\prime}\right)$.

- The contact vertices of $G_{x}^{u}$ (resp. $G_{x}^{n}$ ) represent $k$ occurrences of unnegated (resp. negated) variable $x$ in the equations of $I$. Every equation $x \oplus y \oplus z=b$ from $I, b \in\{0,1\}$, also belongs to $J$ with weight 1 .

This way we produce an instance $J$ of the $w$-MAX-3-LIN-2 problem. Any assignment to variables from $\nu(I)$ generates an assignment to variables of $J$ in a 
natural way: the value of a variable $x$ is assigned to all variables of $G_{x}^{u}$, and the value opposite to $x, \bar{x}=1-x$, is assigned to all vertices of $G_{x}^{n}$. Such assignment to the variables of $J$ is called standard. Similarly to the previous reduction, any assignment to variables of $J$ can be converted to a standard one without increasing the weight of unsatisfied equations as it follows from properties of an amplifier.

\section{$3 \quad$ The weighted bi-wheel amplifiers}

The previous reductions were based on a theoretical model of amplifiers with required properties, without proving their existence. In this section we introduce a class of weighted graphs with such expanding properties that generalise the bi-wheel amplifiers from [9]. Further we describe in the details the properties of the instances of the subproblem of $w$-MAX-3-LIN-2, called the Hybrid bi-wheel instances.

Definition 2. Let an integer $k>0$ and a rational number $\tau>1$ be such that $\tau k$ is an integer. The weighted $(2 k, \tau)$-bi-wheel amplifier $W_{k, \tau}=(V, E)$, $p: E \rightarrow \mathbb{R}^{+}$, is a (weighted) 3-regular amplifier with a specific balanced partition constructed as follows: Take two disjoint cycles, each on $\tau k$ vertices (connected in consecutive order $), V^{u}=\left\{1^{u}, 2^{u}, \ldots,(\tau k)^{u}\right\}$ and $V^{n}=\left\{1^{n}, 2^{n}, \ldots,(\tau k)^{n}\right\}$, respectively. Select the sets of $k$ contacts $D^{u} \subseteq V^{u}$ and $D^{n} \subseteq V^{n}$ as $D^{u}=$ $\left\{c_{1}^{u}, c_{2}^{u}, \ldots, c_{k}^{u}\right\}, D^{n}=\left\{c_{1}^{n}, c_{2}^{n}, \ldots, c_{k}^{n}\right\}$. The remaining vertices of both cycles, $V^{u} \backslash D^{u}$ and $V^{n} \backslash D^{n}$, are checkers.

To complete the construction, consider a perfect matching between the checkers of these two cycles where each matching edge has one vertex in the first cycle $V^{u} \backslash D^{u}$ and another one in the second cycle $V^{n} \backslash D^{n}$.

We assume that in each cycle of the bi-wheel consecutive contacts are separated by a chain of several (at least 1 ) checkers. Hence, in particular, $\tau \geq 2$.

Remark 2. Let us denote by $\mathcal{C}^{u}\left(\mathcal{C}^{n}\right.$, resp.) the set of edges contained in the first (the second, resp.) cycle in $W_{k, \tau}$, so $\mathcal{C}^{u}=\left\{\left\{i^{u},(i+1)^{u}\right\}: i=1,2, \ldots, \tau k\right\}$ and $\left.\mathcal{C}^{n}=\left\{\left\{i^{n},(i+1)^{n}\right\}\right\}: i=1,2, \ldots, \tau k\right\}$ (the vertex $\tau k+1$ is the vertex 1$)$, and by $\mathcal{M} \subseteq E$ the associated perfect matching on the set of checkers. Clearly, $\left|\mathcal{C}^{u}\right|=\left|\mathcal{C}^{n}\right|=\tau k,|\mathcal{M}|=\left|V^{u} \backslash X^{u}\right|=\left|V^{n} \backslash X^{n}\right|=(\tau-1) k$.

In this paper we consider only bi-wheel amplifiers $\left(W_{k, \tau}, p\right)$ whose weights have uniform cycle weight $p_{c}$ for all cycle edges of both $\mathcal{C}^{u}$ and $\mathcal{C}^{n}$, and another uniform matching weight $p_{m}$ for all matching edges from $\mathcal{M}$.

Now we are ready to describe the specific properties of the Hybrid bi-wheel instances of $w$-MAX-3-LIN-2 based on a fixed $(2 k, \tau)$-bi-wheel amplifier $W_{k, \tau}$ with weights $p_{c}$ and $p_{m}$.

Theorem 3. For every $\varepsilon \in\left(0, \frac{1}{4}\right)$ and $b \in\{0,1\}$ there exist instances of w-MAX-3-LIN-2, called Hybrid $\left(W_{k, \tau}, p\right)$, with the following properties: 
(i) each variable of the system equations $\operatorname{Hybrid}\left(W_{k, \tau}, p\right)$ occurs exactly 3 times;

(ii) $m$ equations are of the form $x \oplus y \oplus z=b$, each of weight 1 ;

(iii) $3 \tau m$ equations are of the form $x \oplus y=0$ each of weight $p_{c}$;

(iv) $\frac{3}{2} m(\tau-1)$ equations are of the form $x \oplus y=1$ each of weight $p_{m}$,

for which it is NP-hard to decide whether there is an assignment to the variables that leaves unsatisfied equations of weight at most $\varepsilon m$, or every assignment to the variables leaves unsatisfied equations of weight at least $(0.5-\varepsilon) m$.

The reduction from $\operatorname{Hybrid}\left(W_{k, \tau}, p\right)$, presented later in Section 4, is a gap preserving reduction to TSP parametrised by a $(2 k, \tau)$-bi-heel amplifier with cycle weights $p_{c}$ and matching weights $p_{m}$. The trade-off between parameters $p_{c}, p_{m}$ and $\tau$ is crucial for quality of approximation lower bounds.

Definition 3. We call the triple $\left(p_{c}, p_{m}, \tau\right)$ admissible if for every $k_{0}$ there exists $k \geq k_{0}$ and $a(2 k, \tau)$-bi-wheel that is an amplifier with cycle weights $p_{c}$ and matching weights $p_{m}$.

The bi-wheel amplifiers introduced by Berman and Karpinski [9] are based on the fact that the triple $\left(p_{c}=1, p_{m}=1, \tau=7\right)$ is admissible. This leads to NP-hardness to approximate TSP to within any constant approximation ratio less than $\frac{123}{122}$. They also observed [9] that their proof (of amplification properties) doesn't seem to work with $\tau=6$ instead $\tau=7$. However, there is an opportunity for fine-tuning here if we allow non-integral $\tau$. If, e.g., $90 \%$ of pairs of consecutive contacts in bi-wheel cycles are separated by 6 checkers, and $10 \%$ of such pairs are separated by a chain of 5 checkers only, then the proof of required amplification properties still works. The detailed explanation together with all computations for wheel amplifiers can be found in the paper [2]. The proof for bi-wheels is very similar, so along these lines one can argue that the triple $\left(p_{c}=1, p_{m}=1\right.$, $\tau=6.9$ ) is admissible. This itself would (very modestly) improve on the lower approximation bound for TSP given in [9].

Introducing weighted amplifier graph constructions seems to have paid off even more compared to improvement of parameters for unweighted amplifiers. In this case we have more freedom in fine-tuning the approximation hardness lower bounds obtained in parametric way, if we can prove that bi-wheel amplifiers with certain parameters $\left(p_{c}, p_{m}, \tau\right)$ exist.

Let us explain trade-off between parameters $\left(p_{c}, p_{m}, \tau\right)$ of bi-wheels in a simple scenario with $p_{m}=1$ fixed. Our contribution allows to use weighted amplifiers with $p_{c}<1$ (strengthening of amplifiers) or with $p_{c}>1$ (relaxing of amplifiers). One can achieve amplifiers with $p_{c}<1$ by increasing $\tau$ from $\tau=7$. On the other hand, to relax to $p_{c}>1$ can be achieved with $\tau<7$. These ideas indicate importance to better understand the exact trade-off between $\left(p_{c}, p_{m}, \tau\right)$ triples for bi-wheel amplifiers that provably exist.

In this paper we don't include too many new results on expanding properties of random graphs, we rather demonstrate effectiveness of weighted parametrised amplifiers and address the question of fine-tuning in $\left(p_{c}, p_{m}, \tau\right)$ triples for biwheel amplifiers. We sketch how these ideas will modestly improve known lower bounds for TSP if we allow bi-wheel with $p_{c}<1$. 
Theorem 4. The triple $\left(p_{c}=\frac{1}{2}, p_{m}=1, \tau=11\right)$ is admissible, hence for every large enough $k \geq k_{0}$ there is a $(2 k, 11)$-bi-wheel that is an amplifier with cycle weights $p_{c}=\frac{1}{2}$ and matching weights $p_{m}=1$.

\section{Gap preserving reduction from $\operatorname{Hybrid}\left(W_{k, \tau}, p\right)$ to TSP}

In this section we describe a gap preserving reduction from the system of equations Hybrid $\left(W_{k, \tau}, p\right)$ to the Travelling SALESMAN problem. In the reduction we suppose that all equations of $\operatorname{Hybrid}\left(W_{k, \tau}, p\right)$ with three variables are of the form $x \oplus y \oplus z=0$ to simplify a discussion later (hence Hybrid $\left(W_{k, \tau}, p\right)$ was obtained via reduction from $Q_{0}(\varepsilon, 2 k)$ ). We also introduce a real parameter $\theta>0$ set to $\theta=\frac{1}{\max \left\{1, p_{m}\right\}}$, in order to simultaneously capture different scenarios $p_{m} \leq 1$ and $p_{m}>1$.

The gap preserving reduction is similar to the reduction presented in [9], the main difference is in using a parametrised weighted $(2 k, \tau)$-bi-wheel amplifier $\left(W_{k, \tau}, p\right)$ introduced in Section 3. We use the concept of forced edges introduced by Lampis in [10] (used also in [9]). The idea is based on the observation that we are able to stipulate that some edges, called forced edges, are to be used at least once in any valid tour. It can be achieved by replacing such an edge with a path of many edges of the same total weight. With this trick we may assume without loss of generality that we can force some edges to be used at least once (see [9] for the details). If $u$ and $v$ are vertices that are connected by a forced edge $e$, we write $\{u, v\}_{F}$ or simply $u v_{F}$. The construction contains some forced edges, all other edges in the constructed graph are unforced edges with edge weight 1 .

We start with an instance $I$ of $Q_{0}(\varepsilon, 2 k)$ with $\nu$ variables, $m$ equations of the form $x \oplus y \oplus z=0$ and use the reduction from Section 2 to create an instance $J$ of $\operatorname{Hybrid}\left(W_{k, \tau}, p\right)$. Using the same notation as in Theorem 3 we construct an instance $G[J]$ of TSP in the following way: for each copy $W_{j}:=\left(W_{k, \tau}, p\right)$, $1 \leq j \leq \nu$, of a $(2 k, \tau)$-bi-wheel we construct a subgraph of $G[J]$ :

(i) each variable $x$ of the bi-wheel $W_{j}$, corresponds to a vertex $x$ in the subgraph,

(ii) for each cycle equation $x \oplus y=0$, we create an unforced edge $x y$ with weight 1 .

Now we add the edges among the vertices of 'bi-wheel' subgraphs using two types of gadgets:

- a 3-variable gadget $H^{3 Q}$ :

for each equation $j, 1 \leq j \leq m$, of the form $x \oplus y \oplus z=0$ we add a 3variable gadget $H_{j}^{3 Q}$ connecting the contacts $x, y, z$, where each contact vertex $x, y$, and $z$ is part of its own $(2 k, \tau)$-bi-wheel. Each gadget $H_{j}^{3 Q}$ contains two new vertices $\gamma^{l}, \gamma^{r}$ for every vertex $\gamma \in\{x, y, z\}$ and two additional vertices $e_{j}^{l}$ and $e_{j}^{r}$, see Figure 1 how the vertices are connected. All edges $\left\{\gamma^{\alpha}, \gamma\right\}_{F}$ with $\alpha \in\{r, l\}$ and $\gamma \in\{x, y, z\}$ are forced edges with weight $w\left(\left\{\gamma^{\alpha}, \gamma\right\}_{F}\right)=0.5+p_{c} \theta$. All remaining edges of $H_{j}^{3 Q}$ are unforced with weight 1 .

- a matching gadget $H^{2 M}$ :

for each equation $x_{t}^{u} \oplus x_{q}^{n}=1$ we add a matching gadget $H^{2 M}$ connecting 
the checkers $x_{t}^{u}$ and $x_{q}^{n}$ via two forced edges $\left\{x_{t}^{u}, x_{q}^{n}\right\}_{F}^{1}$ and $\left\{x_{t}^{u}, x_{q}^{n}\right\}_{F}^{2}$, each of the same weight $2 p_{c} \theta$ (Figure 2).

At the end of the construction, we add a new central vertex $s$ that is connected to every gadget $H_{j}^{3 Q}$ with two forced edges $\left\{e_{j}^{l}, s\right\}_{F}$ and $\left\{e_{j}^{r}, s\right\}_{F}$, both with weight $0.5, w\left(\left\{e_{j}^{\alpha}, s\right\}_{F}\right)=0.5$ for both $\alpha \in\{r, l\}$.

Observe that the construction doesn't need gadgets for the cycle edges, the connections between the matching edge gadgets are sufficient to encode these constraints.

bi-wheel $G_{x} \ldots \quad$ bi-wheel $G_{y} \ldots \quad$ bi-wheel $G_{z} \ldots$

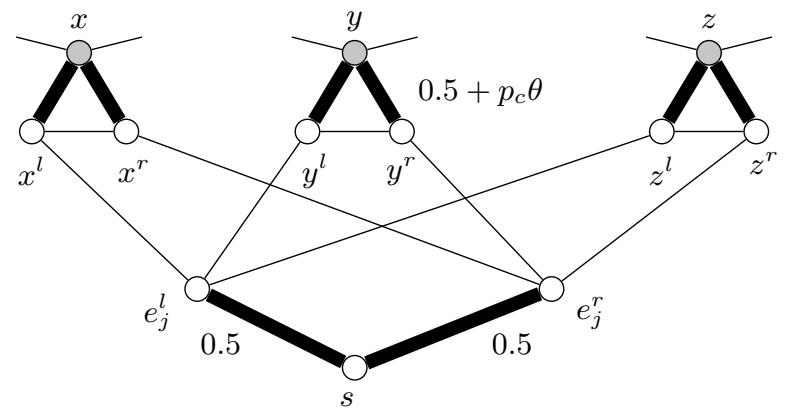

Fig. 1. An example of a 3 -variable gadget $H_{j}^{3 Q}$ including the central vertex $s$, which is not part of the gadget. Thick lines represent forced edges.

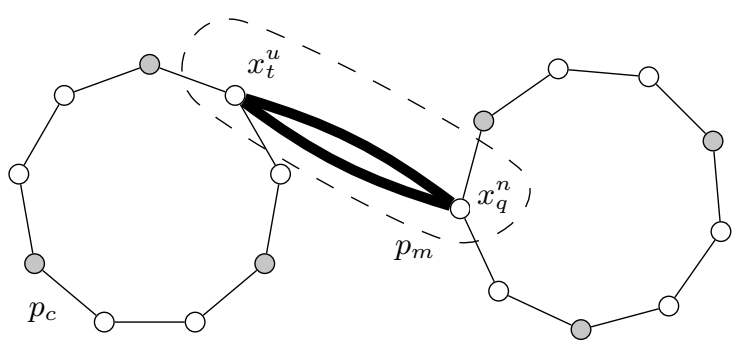

Fig. 2. A gadget $H^{2 M}$ inside the bi-wheel $G_{x}$ for the equations $x_{t}^{u} \oplus x_{q}^{n}=1$ contains only two forced edges, represented as thick lines.

Now in the following we describe in the details the properties of the gap preserving reduction from the Hybrid $\left(W_{k, \tau}, p\right)$ to the Travelling Salesman problem.

Local edge cost. To count the cost $c(T)$ of a tour $T$, we use the local edge cost counting based on the ideas from [9]: the cost $w(u v)$ of any edge $u v$ of $T$ is 
split into two nonnegative parts, one attached to $u$ and the second one to $v$. If an edge $u v$ doesn't contain $s$ then cost is split equally with contribution $0.5 w(u v)$ for each vertex, but for edges of the form $u s$, the full cost contributes to $u$, and none to $s$.

Let $T$ be a multi-set of edges from $E$ that defines a quasi-tour in $G[J](V, E)$. Then for a set $V^{\prime} \subseteq V$, the local edge cost of $V^{\prime}$ is formally defined as

$$
c_{T}\left(V^{\prime}\right)=\sum_{u \in V^{\prime} \backslash\{s\}} \sum_{u v \in T} 0.5 w(u v)+\sum_{e_{j}^{\alpha} \in V^{\prime}} \sum_{e_{j}^{\alpha} s \in T} 0.5 w\left(e_{j}^{\alpha} s\right) .
$$

Note that for two vertex sets $V_{1}, V_{2}$ we have $c_{T}\left(V_{1} \cup V_{2}\right) \leq c_{T}\left(V_{1}\right)+c_{T}\left(V_{2}\right)$ (with equality for disjoint sets), and $c_{T}(V)=\sum_{e \in T} w(e)$.

In Subsection 4.2 we also use the full local cost of the quasi-tour $T$ for the set $V^{\prime}, c_{T}^{*}\left(V^{\prime}\right)$, which is defined as follows: if $\#_{T}\left(V^{\prime}\right)$ is the number of connected components induced by $T$ which are fully contained in $V^{\prime}$, then

$$
c_{T}^{*}\left(V^{\prime}\right)=c_{T}\left(V^{\prime}\right)+2 \#_{T}\left(V^{\prime}\right) .
$$

Intuitively, $c_{T}^{*}\left(V^{\prime}\right)$ captures the cost of the full tour restricted to $V^{\prime}$ : it includes the local edge cost and the cost of a connection of the components on $V^{\prime}$ of the lowest possible price (using two unforced edges), to the rest of the tour.

\subsection{How to construct a tour from an assignment}

Given an instance $J$ of the Hybrid $\left(W_{k, \tau}, p\right)$ and an assignment $\varphi$ to its variables, we describe a construction of a tour $T$ in $G[J]$ with cost related to $\varphi$.

Lemma 1. Let $J$ be an instance of Hybrid $\left(W_{k, \tau}, p\right)$ from Theorem 3. If there exists an assignment $\varphi$ to the variables of $J$ with unsatisfied equations of total weight $\Delta$, then there exists a tour in $G[J]$ with cost at most

$$
\left(\frac{3}{2}(\tau-1)\left(4 p_{c} \theta+1\right)+6 p_{c} \theta+10\right) m+2 \nu+\Delta .
$$

\subsection{How to define an assignment from a tour}

Now we need to prove the opposite direction of the gap preserving reduction: given a tour in $G[J]$ the task is to define an assignment to the variables of the system equations $I$ of $\operatorname{Hybrid}\left(W_{k, \tau}, p\right)$ such that weight of unsatisfied equations is in a correlation with cost of a given tour.

Lemma 2. If there is a tour in $G[J]$ with cost

$$
\left(\frac{3}{2}(\tau-1)\left(4 p_{c} \theta+1\right)+6 p_{c} \theta+10\right) m+\Delta-2,
$$

then there is an assignment to the instance $J$ that leaves unsatisfied equations of weight at most $\Delta \cdot \max \left\{1, p_{m}\right\}=\frac{\Delta}{\theta}$, where $\theta=\frac{1}{\max \left\{1, p_{m}\right\}}$. 
The high-level idea of the proof is to partition the vertex set of $G[J]$ into the gadget-based subgraphs similarly as in the proof of Lemma 1. For each such subgraph we give a lower bound on the local edge cost of any quasi-tour restricted to it, which in fact corresponds to cost of the tour constructed in Lemma 1. If a given quasi-tour behaves inside a gadget differently, its cost must be obviously higher. The difference between the tour's local edge cost and the lower bound is called the credit of the gadget. Based on the tour we define an assignment for $J$ and show that the total sum of credits can be used to bound from above the weight of unsatisfied equation, where the total sum of credits is at most $\Delta$.

Theorem 5. If $\left(p_{c}, p_{m}, \tau\right)$ is an admissible triple then it is NP-hard to approximate the TRAVELLING SALESMAN problem to within any constant approximation ratio less than

$$
1+\frac{1}{3(\tau-1)\left(4 p_{c}+\max \left\{1, p_{m}\right\}\right)+12 p_{c}+20 \max \left\{1, p_{m}\right\}} .
$$

Proof. Let $\varepsilon \in\left(0, \frac{1}{4}\right)$. Consider a $(2 k, \tau)$-bi-wheel with large enough $k$, which is an amplifier with cycle weights $p_{c}$ and matching weights $p_{m}$. We have instances of $\operatorname{Hybrid}\left(W_{k, \tau}, p\right)$ with $\nu$ copies of a bi-wheel $\left(W_{k, \tau}, p\right), m$ equations of the form $x \oplus y \oplus z=0$ each of weight $1,3 \tau \mathrm{r}$ equations of the form $x \oplus y=1$ each of weight $p_{m}$ with the following NP-hard gap results: It is NP-hard to decide whether there is an assignment to the variables that leaves unsatisfied equations of weight at most $\varepsilon m$, or every assignment to the variables leaves unsatisfied equations of weight at least $(0.5-\varepsilon) m$. Due to Lemma 1 and 2 we now know that for produced instances $G[J]$ of TSP it is NP-hard to decide whether there is a tour with cost at most $\left(\frac{3}{2}(\tau-1)\left(4 p_{c} \theta+1\right)+6 p_{c} \theta+10\right) m+2 \nu+\varepsilon m$, where $\theta=\frac{1}{\max \left\{1, p_{m}\right\}}$ or all tours have cost at least $\left(\frac{3}{2}(\tau-1)\left(4 p_{c} \theta+1\right)+6 p_{c} \theta+10\right) m+(0.5-\varepsilon) m \cdot \theta-2$.

The ratio between these two cases can get arbitrarily close to

$$
1+\frac{1}{3(\tau-1)\left(4 p_{c}+\max \left\{1, p_{m}\right\}\right)+12 p_{c}+20 \max \left\{1, p_{m}\right\}}
$$

by appropriate choices of $\varepsilon>0$ and large enough $k$.

Therefore, using the constants of admissible triples from Theorem 4 we can conclude

Corollary 1. It is NP-hard to approximate the TravelLING SALESMAN problem within any constant approximation ratio less than $\frac{117}{116}$.

\section{Conclusion}

The methods of this paper provide a new motivation for the study of expanding properties of random graphs. As we have demonstrated, introducing the parametrised weighted amplifiers and weighted low occurrence ConstrainT SATISFACTION problems as intermediate steps in the NP-hard gap reductions, 
allows more flexibility in fine-tuning their expanding parameters. We show that already slight improvement of known expander values modestly improve the hardness of approximation for TSP from the current best value $\frac{123}{122}([9])$ to the new value $\frac{117}{116}$. The introduced method of weighted amplifiers (or expanders) can be of independent interest. Such technique could be used in the gap preserving reductions for other edge-weighted optimisation problems to improve their approximation hardness results.

\section{References}

1. H. J. Böckenhauer, J. Hromkovič, R. Klasing, S. Seibert and W. Unger, An improved lower bound on the approximability of metric TSP and approximation algorithms for the TSP with sharpened triangle inequality. In Proc. 17th Ann. Symp. on Theoretical Aspects of Comp. Sci. (STACS'00), LNCS 1770, 382-394.

2. M. Chlebík and J. Chlebíková, Approximation Hardness for Small Occurrence Instances of NP-Hard Problems, Proc. of the 5th CIAC, LNCS 2653, 2003, Springer, 152-164 (also ECCC Report TR02-73, 2002).

3. M. Chlebík and J. Chlebíková, The Steiner tree problem on graphs: Inapproximability results, Theoretical Computer Science 406 (2008), 207-214.

4. N. Christofides, Worst-case analysis of a new heuristic for the traveling salesman problem, Technical report, Graduate School of Industrial Administration, CarnegieMellon University, Pittsburgh PA, 1976.

5. L. Engebretsen, An explicit lower bound for TSP with distances one and two, Algorithmica 35(4) (2003), 301-319. Preliminary version in STACS 1999.

6. O. S. Gharan, A. Saberi, and M. Singh, A randomized rounding approach to the traveling salesman problem, IEEE 52nd Annual Symposium on Foundations of Computer Science, FOCS 2011, pp. 550-559.

7. J. Håstad, Some optimal inapproximability results, Journal of ACM 48 (2001), 798-859.

8. M. Held, and R. M. Karp, The traveling-salesman problem and minimum spanning trees, Operations Research 18 (1970), 1138--1162.

9. M. Karpinski, M. Lampis, and R. Schmied, New inapproximability bounds for TSP, Journal of Computer and System Science 81 (2015), 1665-1677.

10. M. Lampis, Improved inapproximability for TSP, Theory Comput. 10(9)(2014), $217-236$.

11. T. Mömke, and O. Svensson, Approximating graphic TSP by matchings. IEEE 52nd Annual Symposium on Foundations of Computer Science, FOCS 2011, pp. $560-569$.

12. M. Mucha, 13/9-approximation for graphic TSP, Theory of Computing Systems $\mathbf{5 5}(4)(2014)$

13. C. H. Papadimitriou and M. Yannakakis: The Traveling Salesman Problem with distances one and two, Math. Oper. Res. 18(1)(1993), 1-11.

14. C. H. Papadimitriou and S. Vempala: On the approximability of the Traveling Salesman Problem, Combinatorica 26(1)(2006), 101-120.

15. A. Sebö and J. Vygen: Shorter tours by nicer ears: 7/5-approximation for the graph-TSP, 3/2 for the path version, and 4/3 for two-edge-connected subgraphs, Combinatorica 34(5)(2014), pp. 597-629.

16. O. Swensson: Overview of New Approaches for Approximating TSP, International Workshop on Graph-Theoretic Concepts in Computer Science, WG 2013, LNCS 8165, pp. 5-11. 